University of New Orleans

ScholarWorks@UNO

7-1979

\title{
Direct relation between Fresnel's interface reflection coefficients for the parallel and perpendicular polarizations
}

\author{
R. M.A. Azzam \\ University of New Orleans, razzam@uno.edu
}

Follow this and additional works at: https://scholarworks.uno.edu/ee_facpubs

Part of the Electrical and Electronics Commons, and the Physics Commons

\section{Recommended Citation}

R. M. A. Azzam, "Direct relation between Fresnel's interface reflection coefficients for the parallel and perpendicular polarizations," J. Opt. Soc. Am. 69, 1007-1016 (1979)

This Article is brought to you for free and open access by the Department of Electrical Engineering at ScholarWorks@UNO. It has been accepted for inclusion in Electrical Engineering Faculty Publications by an authorized administrator of ScholarWorks@UNO. For more information, please contact scholarworks@uno.edu. 


\title{
Direct relation between Fresnel's interface reflection coefficients for the parallel and perpendicular polarizations
}

\author{
R. M. A. Azzam \\ Department of Electrical Engineering, School of Engineering, University of New Orleans, Lakefront, New Orleans, Louisiana \\ 70122
}

(Received 11 December 1978)

\begin{abstract}
We have found a significant relation, $r_{p}=r_{s}\left(r_{s}-\cos 2 \phi\right) /\left(1-r_{s} \cos 2 \phi\right)$, between Fresnel's interface complex-amplitude reflection coefficients $r_{p}$ and $r_{s}$ for the parallel $(p)$ and perpendicular $(s)$ polarizations at the same angle of incidence $\phi$. This relation is universal in that it applies to reflection at all interfaces between homogeneous isotropic media collectively and, of course, throughout the electromagnetic spectrum. We investigate the properties of this function, $r_{p}=f\left(r_{s}\right)$, and its inverse, $r_{s}=g\left(r_{p}\right)$, as conformal mappings between the complex planes of $r_{s}$ and $r_{p}$. A related function, $\rho=\left(r_{s}-\cos 2 \phi\right) /\left(1-r_{s} \cos 2 \phi\right)$, which is a bilinear transformation, is also studied, where $\rho=r_{p} / r_{s}$ is the (ellipsometric) ratio of reflection coefficients. Several previously described reflection characteristics come out readily as specific results of this work. Simple explicit analytical and graphical solutions are provided to determine reflection phase shifts and the dielectric function from measured $p$ and $s$ reflectances at the same angle of incidence. We also show that when $r_{p}$ is real, negative, and in the range $-1 \leq r_{p} \leq-\tan ^{2}\left(\phi-45^{\circ}\right), r_{s}$ is complex and its locus in the complex plane is an arc of a circle with center on the real axis at sec2 $\phi$ and radius of $|\tan 2 \phi|$. Under these conditions, we also find the interesting result that $\left|r_{s}\right|=\left|r_{p}\right|^{1 / 2}$.
\end{abstract}

\section{INTRODUCTION}

The reflection of a monochromatic electromagnetic plane wave at the planar interface between two linear homogeneous and isotropic media is governed by the well-known Fresnel coefficients. For the two linear polarizations parallel ( $p$ or TM) and perpendicular ( $s$ or TE) to the plane of incidence, these coefficients are given by ${ }^{1}$

$$
\begin{aligned}
r_{p} & =\frac{\epsilon \cos \phi-\left(\epsilon-\sin ^{2} \phi\right)^{1 / 2}}{\epsilon \cos \phi+\left(\epsilon-\sin ^{2} \phi\right)^{1 / 2}}, \\
r_{s} & =\frac{\cos \phi-\left(\epsilon-\sin ^{2} \phi\right)^{1 / 2}}{\cos \phi+\left(\epsilon-\sin ^{2} \phi\right)^{1 / 2}},
\end{aligned}
$$

where $\phi$ is the angle of incidence and $\epsilon$ is the ratio of the dielectric function of the medium of refraction to that of the medium of incidence. We assume the $e^{j \omega t}$ time dependence and $p$ and $s$ directions as in the Nebraska (Muller) conventions. ${ }^{2}$

In this paper we find a direct relation between $r_{p}$ and $r_{s}$ at the same angle of incidence $\phi$, study its properties, and give one of its applications. This complements earlier work on the transformation of the Fresnel coefficients between normal and oblique incidence. ${ }^{3}$

\section{RELATION BETWEEN $r_{p}$ AND $r_{s}$}

To relate $r_{p}$ and $r_{s}$ we eliminate $\epsilon$ between Eqs. (1) and (2). In terms of

$$
x=\left(\epsilon-\sin ^{2} \phi\right)^{1 / 2},
$$

Eqs. (1) and (2) become

$$
\begin{aligned}
r_{p} & =\frac{\cos \phi\left(x^{2}+\sin ^{2} \phi\right)-x}{\cos \phi\left(x^{2}+\sin ^{2} \phi\right)+x}, \\
r_{s} & =(\cos \phi-x) /(\cos \phi+x) .
\end{aligned}
$$

From Eq. (5), $x=\cos \phi\left(1-r_{s}\right) /\left(1+r_{s}\right)$, which we substitute into Eq. (4) to obtain

$$
r_{p}=r_{s}\left(r_{s}-\cos 2 \phi\right) /\left(1-r_{s} \cos 2 \phi\right) .
$$

Equation (6) is the desired direct relation between Fresnel's interface complex-amplitude reflection coefficients $r_{p}$ and $r_{s}$ for the $p$ and $s$ polarizations at the same angle of incidence 4 $\phi$. Significantly, this relation is independent of the two media that define the interface and applies, of course, throughout the entire electromagnetic spectrum. Although Eqs. (1) and (2), hence Eq. (6), are valid generally when both media are absorbing and $\phi$ is complex, we will assume hereafter that the medium of incidence is transparent and that $\phi$ is real.

At normal incidence, $\phi=0$, and at grazing incidence, $\phi=$ $90^{\circ}$, Eq. (6) reduces to $r_{p}=-r_{s}$ and $r_{p}=r_{s}$ respectively, as expected. When $\phi=45^{\circ}$, Eq. (6) reduces to $r_{p}=r_{s}^{2}$, a result which is also known to be true for this special case. ${ }^{5,6,7}$

Equation (6) predicts Brewster's condition, namely that $r_{p}$ can become zero; this occurs when

$$
r_{s}=\cos 2 \phi,
$$

which is a simple expression for the nonzero reflection coefficient for the $s$ polarization at the Brewster angle $\left(\phi=\phi_{B}\right)$.

The partial derivative of Eq. (6) with respect to $r_{s}$,
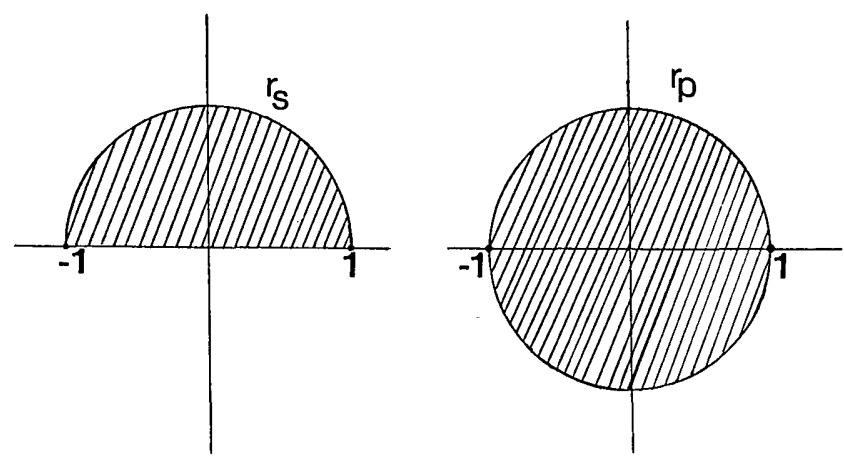

FIG. 1. Domains of all permissible values of Fresnel's interface reflection coefficients $r_{s}$ (left) and $r_{p}$ (right) for the $s$ and $p$ polarizations. 

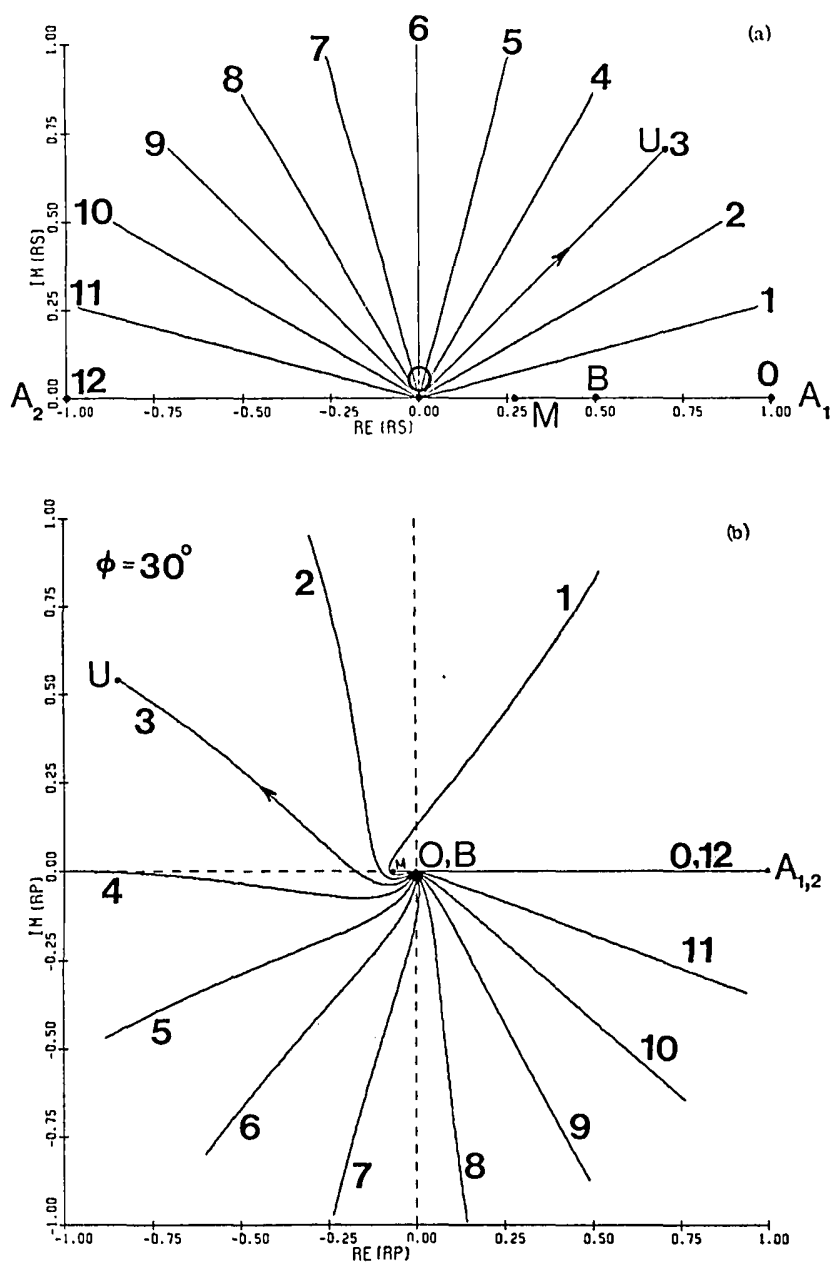

FIG. 2. Mapping of $r_{s}$ onto $r_{p}$ according to the complex analytic function $r_{p}=r_{s}\left(r_{s}-\cos 2 \phi\right) /\left(1-r_{s} \cos 2 \phi\right)$ when $\phi=30^{\circ}$. The angularly equispaced straight lines through the origin $0,1,2, \ldots, 12$ in the $r_{s}$ plane represent lines of equal reflection phase shift for the $s$ polarization, $\delta_{s}=\operatorname{argr}_{s}$ $=0,15^{\circ}, 30^{\circ}, \ldots, 180^{\circ}$ respectively and their images in the $r_{p}$ plane are marked by the same numbers. Points that are images of one another are also marked by the same letters. (This notation applies to the following figures as well.) As $r_{s}$ scans straight line 3 , for example, from the origin $O$ to $U$ on the unit circle in the $r_{s}$ plane, $r_{p}$ scans curve 3 from the origin $O$ to $U$ on the unit circle in the $r_{p}$ plane, in the direction of the indicated arrows.

$$
\frac{\partial r_{p}}{\partial r_{s}}=\left(-r_{s}^{2} \cos 2 \phi+2 r_{s}-\cos 2 \phi\right) /\left(1-r_{s} \cos 2 \phi\right),^{2}
$$

vanishes when 8

$$
r_{s}=\sec 2 \phi-\tan 2 \phi=-\tan \left(\phi-45^{\circ}\right) .
$$

Substitution of this value of $r_{s}$ into Eq. (6) gives

$$
r_{p}=-(\sec 2 \phi-\tan 2 \phi)^{2}=-\tan ^{2}\left(\phi-45^{\circ}\right)=-r_{s}^{2} \text {. }
$$

The reader can verify that Eqs. (9) and (10) give the Fresnel coefficients for reflection at interfaces between transparent media such that the angle of refraction is $45^{\circ}$. Because $\partial r_{p} / \partial r_{s}=\left(\partial r_{p} / \partial \epsilon\right)\left(\partial \epsilon / \partial r_{s}\right)$, it follows that $\partial r_{p} / \partial \epsilon=\partial r_{p} / \partial \nu$ $=0$, where $\epsilon=\nu^{2}$ and $\nu$ is the complex relative refractive index. This result is in agreement with what we have found before in Ref. 9.

In the following section we investigate Eq. (6) more fully as a conformal mapping ${ }^{10}$ between the complex planes of $r_{s}$ and $r_{p}{ }^{11}$

\section{MAPPING OF $r_{s}$ ONTO $r_{p}$}

In the Nebraska (Muller) conventions, ${ }^{2} r_{s}$ is restricted to the inside and boundaries of the upper-half of the unit circle in the complex $r_{s}$ plane, and, consequently from Eq. (6), $r_{p}$ is limited to the interior and boundary of the full unit circle in the complex $r_{p}$ plane, Fig. 1. For every value of $r_{s}$ only one value of $r_{p}$ is obtained from Eq. (6), but not vice versa. There is one-to-one correspondence between points in the domains of all permissible values of $r_{s}$ and $r_{p}$ shown in Fig. 1 except for points along the real axis.

Figure 2 shows mapping by the complex analytic function $r_{p}=f\left(r_{s}\right)$ of Eq. (6) of angularly equispaced straight lines through the origin of the $r_{s}$ plane (lines of equal reflection phase shift for the $s$ polarization $\delta_{s}=\arg r_{s}=0,15^{\circ}, 30^{\circ}, \ldots$,
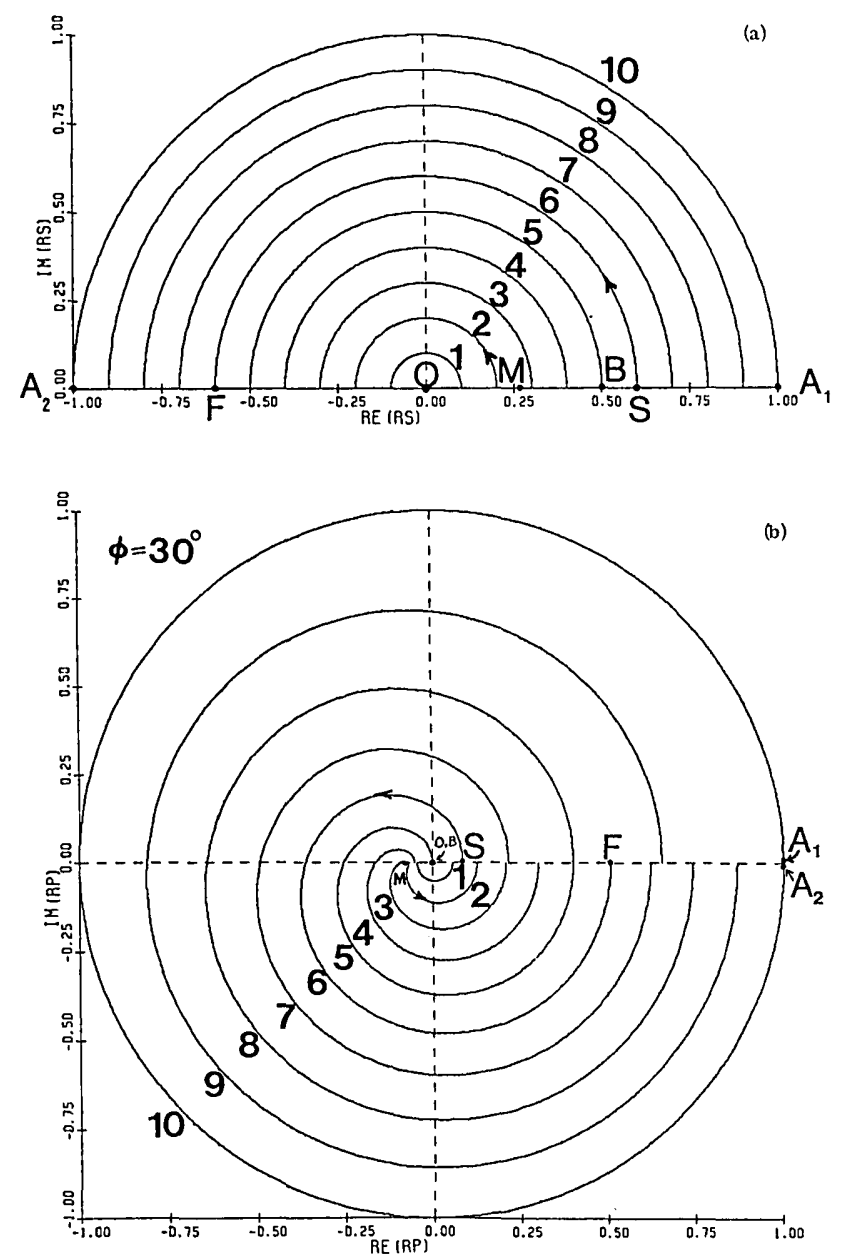

FIG. 3. Mapping of $r_{s}$ onto $r_{p}$ according to the complex analytic function $r_{p}=r_{s}\left(r_{s}-\cos 2 \phi\right) /\left(1-r_{s} \cos 2 \phi\right)$ when $\phi=30^{\circ}$. The equispaced semicircles centered on the origin $1,2,3, \ldots, 10$ in the $r_{s}$ plane represent lines of equal amplitude reflectance for the $s$ polarization, $\left|r_{s}\right|=0.1,0.2$, $0.3, \ldots, 1.0$ respectively and their images in the $r_{p}$ are marked by the same numbers. The images in the $r_{p}$ plane of semicircles 1,2 lie entirely below the real axis, while the images of semicircles 3 to 10 are one-full-revolution spirals. As $r_{s}$ traces semicircle 6 , for example, from $S$ to $F$ in the $r_{s}$ plane, $r_{p}$ traces spiral 6 from $S$ to $F$ in the $r_{p}$ plane, in the direction of the indicated arrows. 
$180^{\circ}$ ) onto the $r_{p}$ plane, for an angle of incidence $\phi=30^{\circ}$. Here, and in other figures, lines and points that are images of one another are marked by the same numbers and letters respectively, and arrows indicate directions in which lines are traced. Mapping of points along the real axis of the $r_{s}$ plane onto the $r_{p}$ plane requires special attention. Such points represent reflection of electromagnetic radiation at interfaces between transparent media. When $r_{s}$ moves along the positive real axis from the origin $O\left(r_{s}=0\right)$ to $M\left(r_{s}=-\tan \left(\phi-45^{\circ}\right)\right.$ $=0.268)$ to $B\left(r_{s}=\cos 2 \phi=0.5\right), r_{p}$ moves along the negative real axis from $O\left(r_{p}=0\right)$ to $M\left(r_{p}=-\tan ^{2}\left(\phi-45^{\circ}\right)=-0.072\right)$
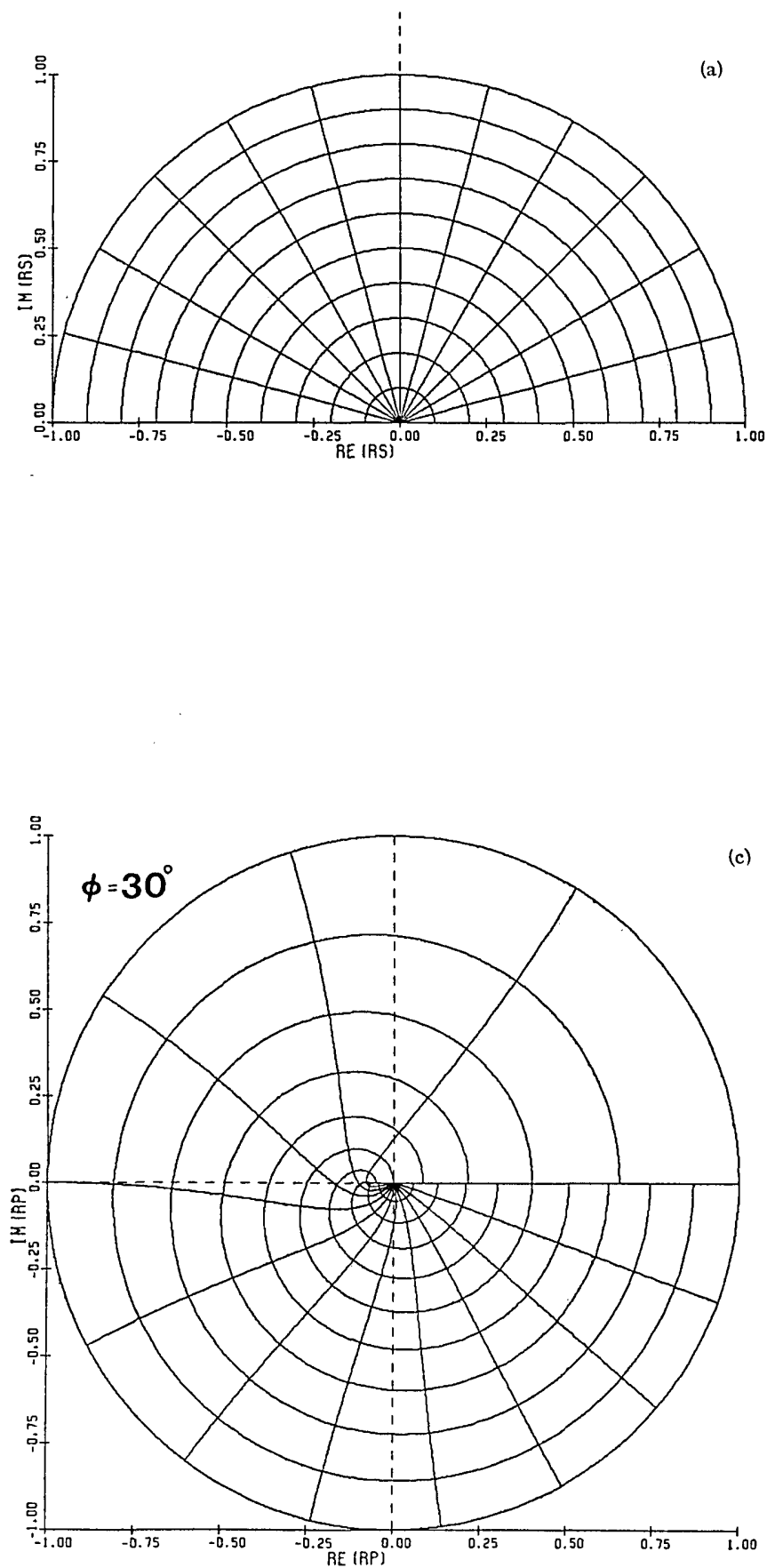

and back to $B$ which coincides with the origin $O$. The reversal of the direction of motion of $r_{p}$ at the point $M$ is consistent with the fact that $\partial r_{p} / \partial r_{s}=0$ at that point [see the discussion associated with Eqs. (9) and (10)]. $M$ represents refraction at $45^{\circ}$ while $B$ represents reflection at the Brewster angle at interfaces between transparent media. If $r_{s}$ moves from $B$ to $A_{1}\left(r_{s}=1\right)$, or from $O$ to $A_{2}\left(r_{s}=-1\right), r_{p}$ moves from $O$ to $A_{1,2}\left(r_{p}=1\right)$. It is evident that two values of $r_{s}$ produce the same value of $r_{p}$.

Figure 3 shows how a family of equispaced semicircles
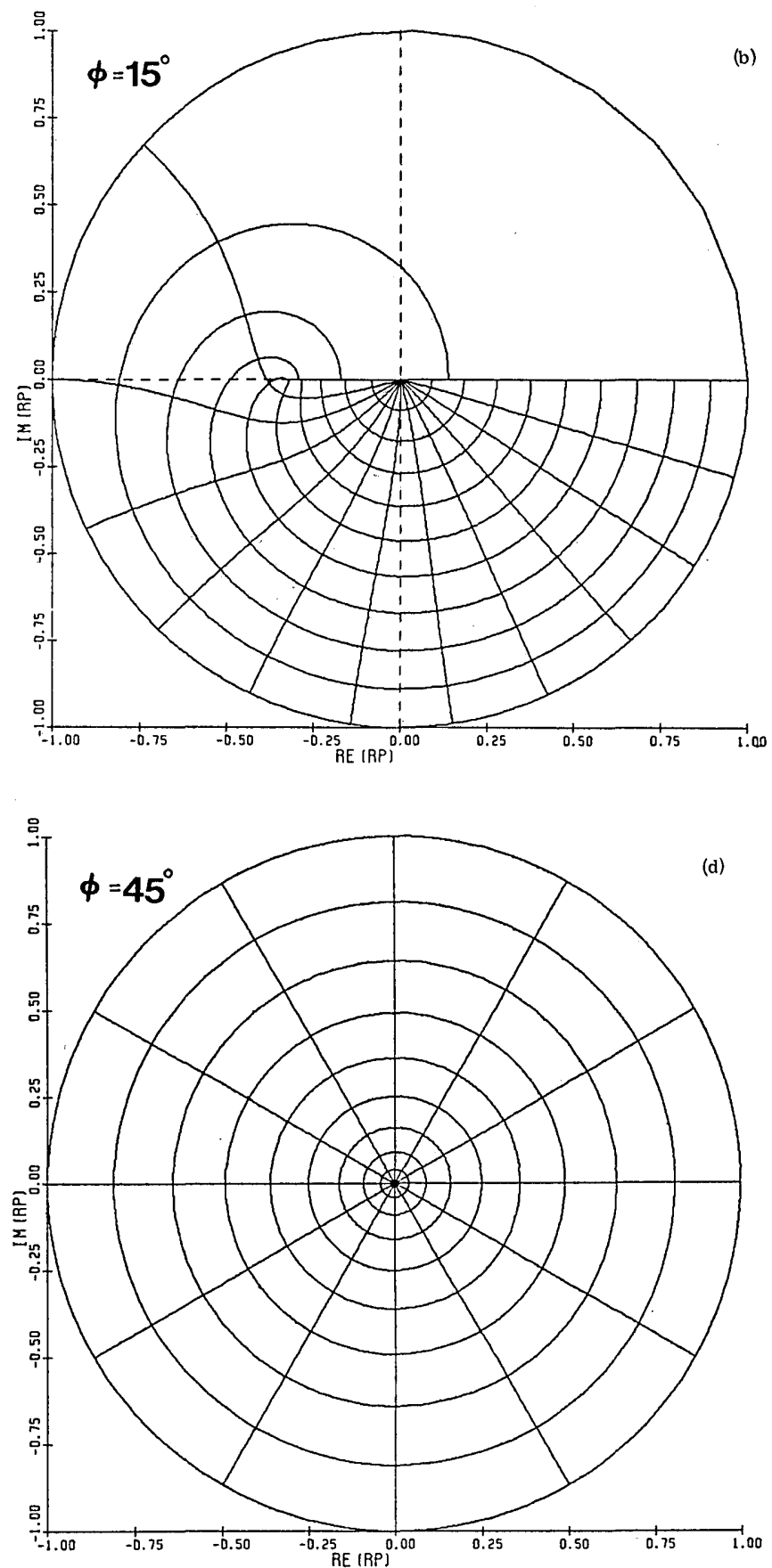

FIG. 4. Mapping of $r_{s}$ onto $r_{p}$ according to the complex analytic function $r_{p}=r_{s}\left(r_{s}-\cos 2 \phi\right) /\left(1-r_{s} \cos 2 \phi\right)$ when $\phi=15^{\circ}, 30^{\circ}, 45^{\circ}, 60^{\circ}, 75^{\circ}$. The orthogonal families of straight lines and semicircles through and around the origin in the $r_{s}$ plane are mapped onto orthogonal sets of curves in the $r_{p}$ plane. The orthogonal sets that correspond to $\phi=30^{\circ}$ are obtained from the superposition of Figs. 2 and 3 . To identify individual curves use Figs. 2 and 3 as a guide. (Continued on p. 1010.) 

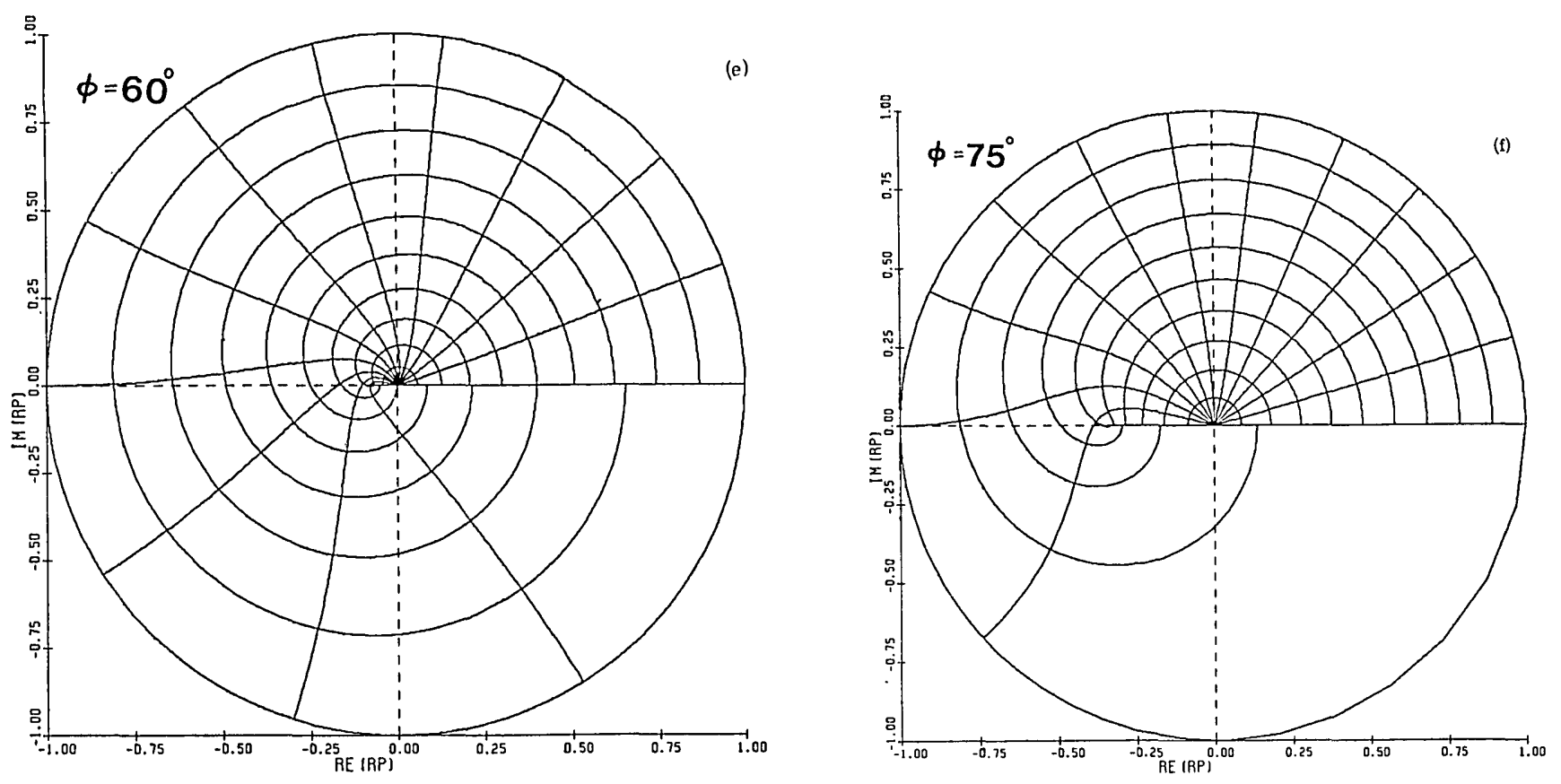

FIG. 4. (continued).

centered on the origin in the $r_{s}$ plane (lines of equal amplitude reflectance for the $s$ polarization $\left|r_{s}\right|=0.1,0.2,0.3, \ldots, 1.0$ ) are mapped by Eq. (6) onto the $r_{p}$ plane, at the same angle of incidence $\phi=30^{\circ}$ as in Fig. 2. Semicircles 1 and 2 have images that lie entirely below the real axis of the $r_{p}$ plane. We can show that this actually applies to all semicircles with radii $\left|r_{s}\right|$ $\leq O M=\left|\tan \left(\phi-45^{\circ}\right)\right|=0.268$. Semicircles 3 to 10 , for which $\left|r_{s}\right|>\left|\tan \left(\phi-45^{\circ}\right)\right|$, have images in the $r_{p}$ plane that are one-full-revolution spirals. The spiralling takes place around the point $M$.

The superposition of Figs. 2 and 3 produces orthogonal families of curves in the $r_{s}$ and $r_{p}$ planes. This is shown in Fig. 4 for five angles of incidence including $30^{\circ}\left(\phi=15^{\circ}, 30^{\circ}, 45^{\circ}\right.$, $60^{\circ}, 75^{\circ}$ ). Orthogonal sets of curves for $\phi=45^{\circ}+\theta$ and $\phi=$ $45^{\circ}-\theta$ are mirror images of one another with respect to the real axis. Figure 4 gives as complete a picture of the mapping properties of Eq. (6) as is graphically possible. It provides useful nomograms that can be used to obtain quick estimates of the complex reflection coefficient $r_{p}$ for given values of $r_{s}$, by locating in the $r_{p}$ plane the point of intersection of the two contours representing $\left|\dot{r}_{s}\right|=$ constant and $\arg r_{s}=$ constant.

\section{INVERSE TRANSFORMATION: MAPPING OF $r_{p}$ ONTO $r_{s}$}

Equation (6) can be inverted easily to give $r_{s}$ as a function of $r_{p}$ :

$$
r_{s}=\frac{1}{2} \cos 2 \phi\left(1-r_{p}\right)+\left(r_{p}+\frac{1}{4} \cos ^{2} 2 \phi\left(1-r_{p}\right)^{2}\right)^{1 / 2} .
$$

The inverse function, $r_{s}=g\left(r_{p}\right)$, Eq. (11), is double-valued; for every $r_{p}$ there are two values of $r_{s}$ that correspond to the two values of the square root. However, because $r_{s}$ is limited to the upper-half of the unit circle, values of $r_{s}$ with negative imaginary parts are rejected. It follows that Eq. (11) gives two physically acceptable values of $r_{s}$ only if $r_{p}$ is real.

Figure 5 shows mapping by the complex analytic function $r_{s}=g\left(r_{p}\right)$ of Eq. (11) of angularly equispaced straight lines through the origin of the $r_{p}$ plane (lines of equal reflection phase shift for the $p$ polarization $\delta_{p}=\arg r_{p}=0,15^{\circ}, 30^{\circ}, \ldots$, $345^{\circ}$ ) onto the $r_{s}$ plane when the angle of incidence $\phi=30^{\circ}$. The origin $r_{p}=0$ represents two physically distinguishable situations: (i) Reflection from interfaces in the limit when the two media on opposite sides of the interface become the same; in this case, we also have $r_{s}=0$, and the situation is represented by the point $O$. (ii) Reflection at the Brewster angle in which case $r_{s}=\cos 2 \phi$, and this instance is represented by the point $B$. Thus the origin of the $r_{p}$ plane has two separate image points in the $r_{s}$ plane one is $O\left(r_{s}=0\right)$ and the other is $B\left(r_{s}=\cos 2 \phi\right)$. When $r_{p}$ moves along the positive real axis of the $r_{p}$ plane from the origin to $A_{1}$ its two distinct images in the $r_{s}$ plane move in opposite directions along the segments of the real axis from $B$ to $A_{1}\left(r_{s}=1\right)$ and from $O$ to $\dot{A}_{1}\left(r_{s}=-1\right)$. If $r_{p}$ moves along the negative real axis of the $r_{p}$ plane from the origin to $M\left(r_{p}=-\tan ^{2}\left(\phi-45^{\circ}\right)=-0.072\right)$ its two images in the $r_{s}$ plane move in opposite directions on the segments of the real axis from $B$ to $M\left(r_{s}=-\tan (\phi-45)\right.$ $=0.268$ ) and from $O$ to $M$. Further movement of $r_{p}^{-}$along the negative real axis of the $r_{p}$ plane from $M$ to $A_{2}$ generates the curve $M A_{2}$ in the $r_{s}$ plane. The interesting conclusion follows immediately that the reflection coefficient of the $p$ polarization can become real and negative while, at the same time, the reflection coefficient for the $s$ polarization is complex. This result, which is readily obtained from the present study, was recently arrived at in a different way. ${ }^{12}$ In the appendix, we prove that the curve $M A_{2}$ in Fig. 5 is in fact an arc of a circle with center on the real axis at $\sec 2 \phi$ and radius of $|\tan 2 \phi|$. This appendix gives other new information that supplements the results of Ref. 12 .

In Fig. 5, straight lines that start from the origin and ter- 

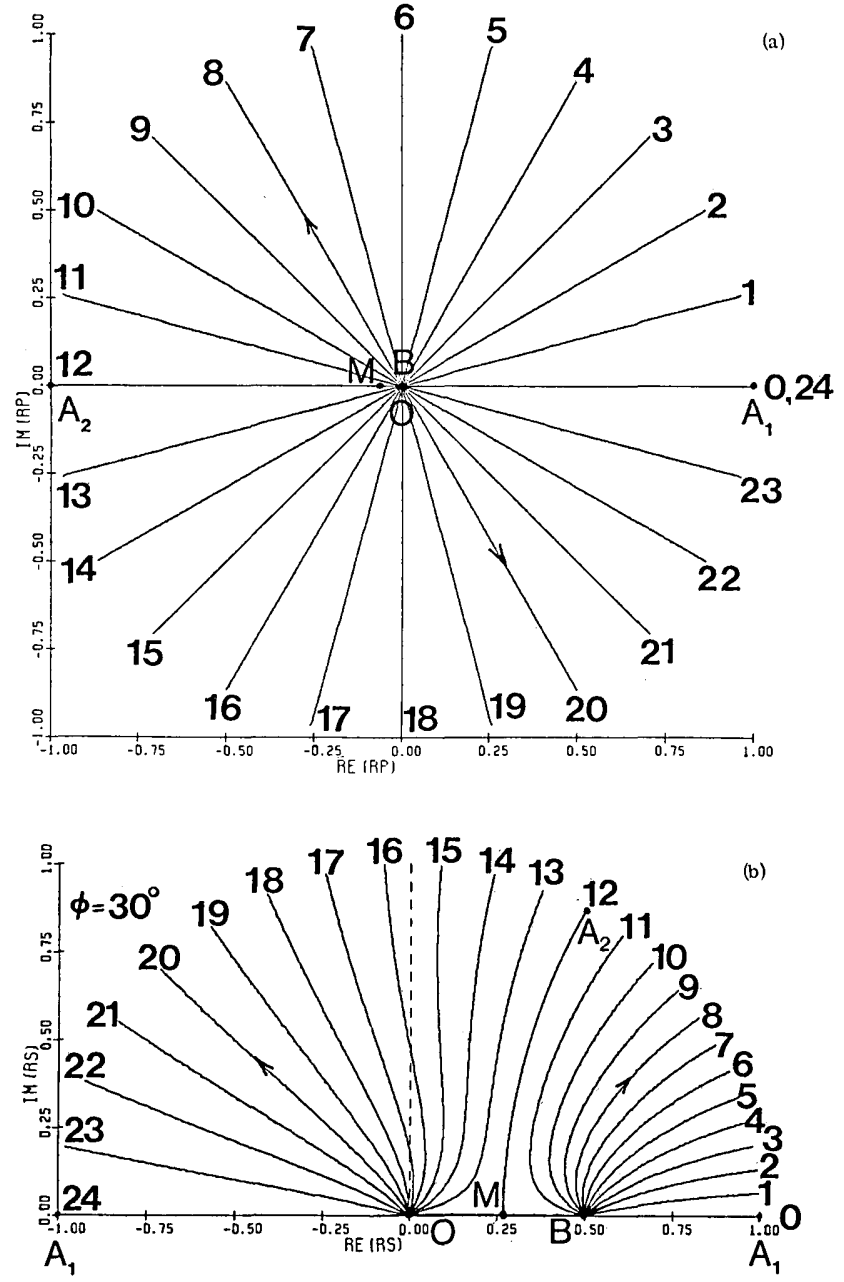

FIG. 5. Inverse mapping of $r_{p}$ onto $r_{s}$ according to the complex analytic function $r_{s}=(1 / 2) \cos 2 \phi\left(1-r_{p}\right)+\left[r_{p}+(1 / 4) \cos ^{2} 2 \phi\left(1-r_{p}\right)^{2}\right]^{1 / 2}$ when $\phi$ $=30^{\circ}$. The angularly equispaced straight lines through the origin $0,1,2$, $\ldots, 23$ in the $r_{p}$ plane represent lines of equal reflection phase shift for the $p$ polarization, $\delta_{p}=\arg r_{p}=0,15^{\circ}, 30^{\circ} \ldots, 345^{\circ}$ respectively and their images in the $r_{s}$ plane are marked by the same numbers. The images in the $r_{s}$ plane of straight lines, $1,2, \ldots, 11$ in the upper half of the $r_{p}$ plane all originate from the point $B\left(r_{s}=\cos 2 \phi=0.5\right)$, while the images of straight lines $13,14, \ldots, 23$ in the lower half of the $r_{p}$ plane all pass through the origin 0 . The segment $M A_{2}$ of the negative real axis of the $r_{p}$ plane is imaged onto circle arc $M A_{2}$ in the $r_{s}$ plane. $M A_{2}$ divides the upper half of the unit circle in the $r_{s}$ plane into two domains, one to its right and the other to its left, that correspond to the upper and lower halves of the unit circle in the $r_{p}$ plane respectively.

minate on the unit circle in the upper half of the $r_{p}$ plane $(0$ $\left.<\arg r_{p}<180^{\circ}\right)$ are mapped onto curves that all originate from the same point $B$ and terminate on (the $\operatorname{arc} A_{1} A_{2}$ of) the unit circle in the $r_{s}$ plane. The remaining straight lines through the origin in the lower half of the $r_{p}$ plane $\left(180^{\circ}<\arg \right.$ $r_{p}<360^{\circ}$ ) are mapped onto a separate set of curves that originate from the point $O$ and terminate on (the $\operatorname{arc} A_{2} A_{1}$ of) the unit circle in the $r_{s}$ plane. Thus the upper and lower halves of the unit circle in the $r_{p}$ plane are mapped onto the subdomains of the upper half of the unit circle in the $r_{s}$ plane to the right and to the left, respectively, of the circle arc $M A_{2}$.

Figure 6 shows how a family of circles centered on the origin in the $r_{p}$ plane (lines of equal amplitude reflectance for the $p$ polarization, $\left.\left|r_{p}\right|=0.1,0.2, \ldots, 1.0\right)$ are mapped by Eq. (11) onto the $r_{s}$ plane.

The superposition of Figs. 5 and 6 produces orthogonal sets of curves in the $r_{p}$ and $r_{s}$ planes. This is shown in Fig. 7 for five angles of incidence including $30^{\circ}\left(\phi=15^{\circ}, 30^{\circ}, 45^{\circ}, 60^{\circ}\right.$, $\left.75^{\circ}\right)$. The image of a circle $\left|r_{p}\right|=$ constant in the $r_{p}$ plane is split into two separate branches in the $r_{s}$ plane when the condition $\left|r_{p}\right| \leq \tan ^{2}\left(\phi-45^{\circ}\right)$ is satisfied. This appears in Fig. 7 for the cases of $\phi=15^{\circ}$ and $\phi=75^{\circ}$. Mirror reflection in the imaginary axis of the orthogonal sets of curves for $\phi$ $=45^{\circ}-\theta$ produces the sets that correspond to $\phi=45^{\circ}+\theta$. Figure 7 provides useful nomograms for finding $r_{s}$ for given values of $r_{p}$.

\section{SIMPLER TRANSFORMATION: MAPPING OF $r_{s}$ ONTO $\rho=r_{p} / r_{s}$}

The ratio of reflection coefficients of the $p$ and $s$ polarizations

$$
\rho=r_{p} / r_{s}
$$

is significant because it can be determined experimentally
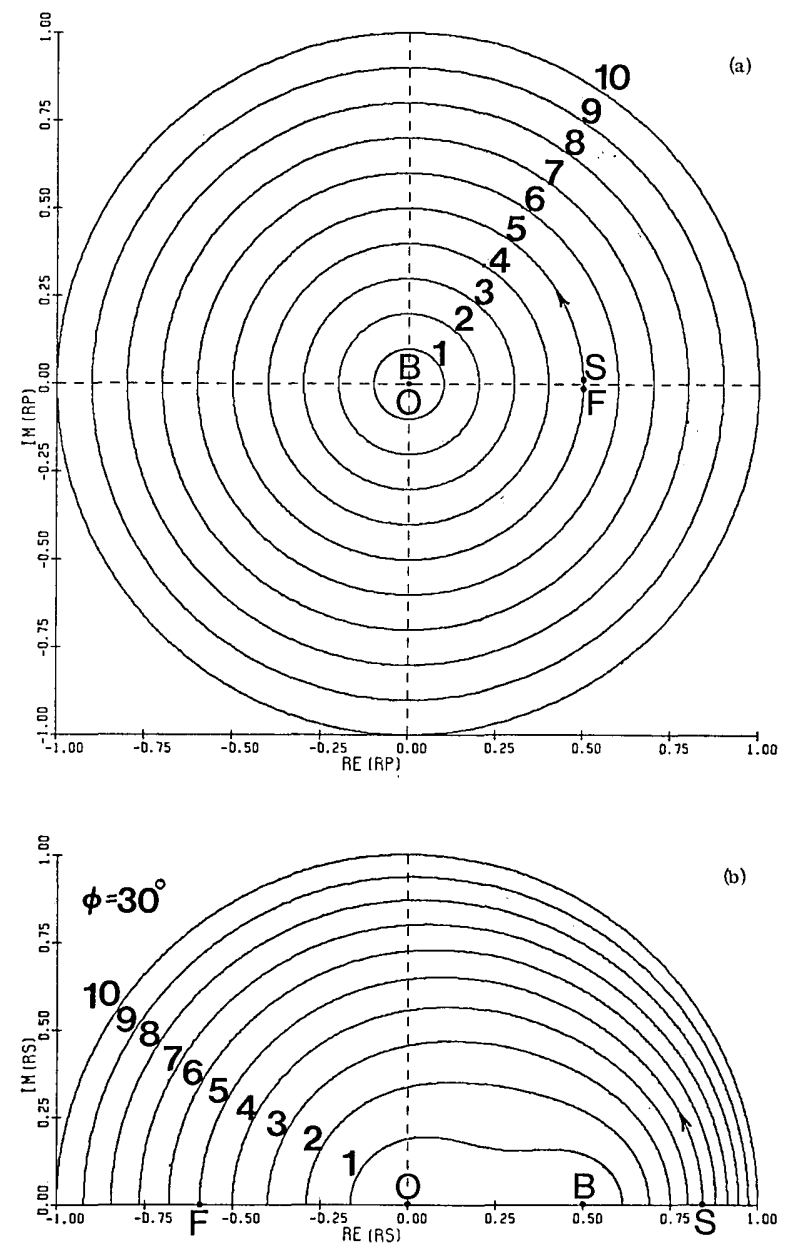

FIG. 6. Inverse mapping of $r_{p}$ onto $r_{s}$ according to the complex analytic function $r_{s}=(1 / 2) \cos \phi\left(1-r_{p}\right)+\left[r_{p}+(1 / 4) \cos ^{2} 2 \phi\left(1-r_{p}\right)^{2}\right]^{1 / 2}$ when $\phi$ $=30^{\circ}$. The equispaced circles centered on the origin $1,2,3, \ldots, 10$ in the $r_{p}$ plane represent lines of equal amplitude reflectance for the $p$ polarization $\left|r_{p}\right|=0.1,0.2,0.3, \ldots, 1.0$ respectively and their images in the $\left|r_{s}\right|$ plane are marked by the same numbers. 

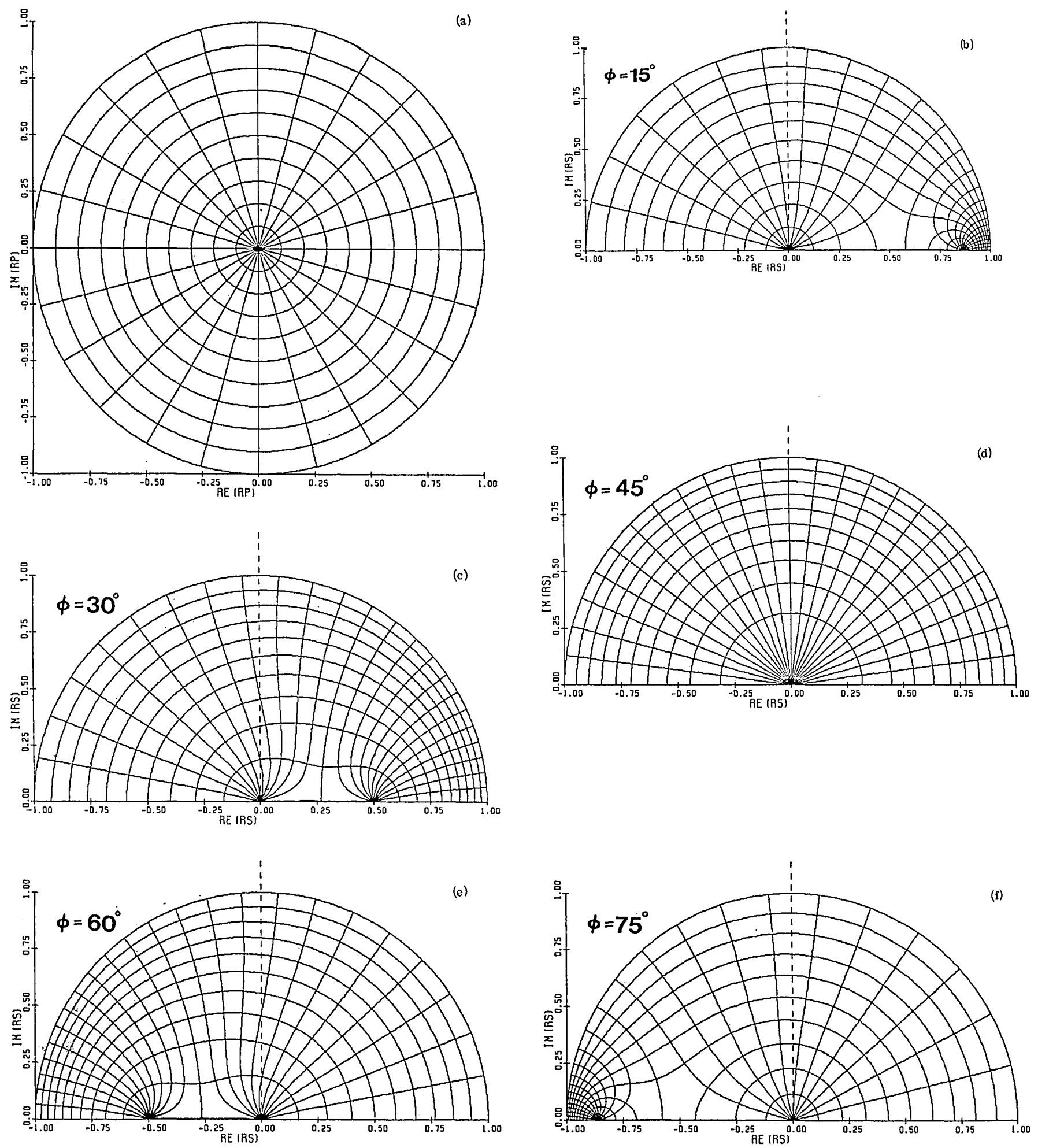

FIG. 7. Inverse mapping of $r_{p}$ onto $r_{s}$ according to the complex analytic function $r_{s}=(1 / 2) \cos 2 \phi\left(1-r_{p}\right)+\left[r_{p}+(1 / 4) \cos ^{2} 2 \phi\left(1-r_{p}\right)^{2}\right]^{1 / 2}$ when $\phi=15^{\circ}$, $30^{\circ}, 45^{\circ}, 60^{\circ}, 75^{\circ}$. The orthogonal families of straight lines and circles through and around the origin in the $r_{p}$ plane are mapped onto orthogonal sets of curves in the $r_{s}$ plane. The orthogonal sets that correspond to $\phi=30^{\circ}$ are obtained from the superposition of Figs. 5 and 6 . To identify individual curves use Figs. 5 and 6 as a guide.

from polarization measurements, i.e. by ellipsometry. ${ }^{13}$ From Eq. (6) we find that

$$
\rho=\left(r_{s}-\cos 2 \phi\right) /\left(1-r_{s} \cos 2 \phi\right) .
$$

Equation (13) indicates that $\rho$ is related to $r_{s}$ via a bilinear
(Möbius) transformation. ${ }^{10} \quad r_{s}$ and $\rho$ are both limited to the upper half of the unit circle in the complex plane.

A bilinear transformation has the distinction of mapping circles (including straight lines which are degenerate circles) in the complex plane of one variable onto circles in the com- 

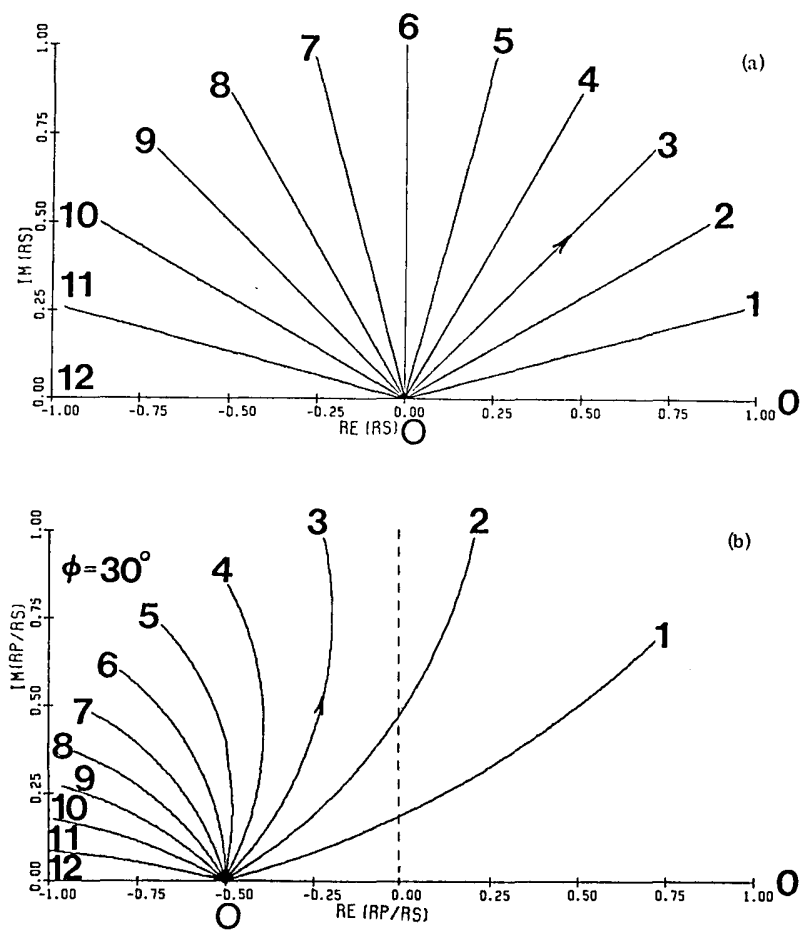

FIG. 8. Mapping of $r_{s}$ onto $\rho=r_{p} / r_{s}$ according to the bilinear transformation $\rho=\left(r_{s}-\cos 2 \phi\right) /\left(1-r_{s} \cos 2 \phi\right)$ when $\phi=30^{\circ}$. Straight lines through the origin in the $r_{s}$ plane, $\delta_{s}=\arg r_{s}=0,15^{\circ}, 30^{\circ}, \ldots, 180^{\circ}$, are mapped onto arcs of coaxial circles that pass through the points $\rho=$ $-\cos 2 \phi$ and $-\sec 2 \phi$ (not shown) in the $\rho$ plane.

plex plane of the other variable. Figure 8 shows how straight lines through the origin of the $r_{s}$ plane $\left(\arg r_{s}=0,15^{\circ}, \ldots\right.$, $180^{\circ}$ ) are mapped onto arcs of coaxial circles in the $\rho$ plane that pass through the common points $\rho=-\cos 2 \phi$ inside the unit circle, and $\rho=-\sec 2 \phi$ outside the unit circle (not shown in Fig. 8). These two points are the images of $r_{s}=0$ and $r_{s}=$ $\infty$, respectively.

Figure 9 shows the mapping of semicircles centered on the origin of the $r_{s}$ plane $\left(\left|r_{s}\right|=0.1,0.2, \ldots, 1.0\right)$ onto semicircles of coaxial circles in the $\rho$ plane that enclose the point $\rho=$ $-\cos 2 \phi$ (which is the image of $r_{s}=0$ ).

The superposition of Figs. 8 and 9 produces orthogonal families of straight lines and semicircles in the $r_{s}$ plane and orthogonal families of circular arcs and semicircles in the $\rho$ plane. This is shown in Fig. 10 for five angles of incidence including $30^{\circ}\left(\phi=15^{\circ}, 30^{\circ}, 45^{\circ}, 60^{\circ}, 75^{\circ}\right)$. Here also mirror reflection in the imaginary axis relates the orthogonal sets at $\phi=45^{\circ}+\theta$ and $45^{\circ}-\theta$.

The inverse of Eq. (13),

$$
r_{s}=(\rho+\cos 2 \phi) /(1+\rho \cos 2 \phi),
$$

gives $r_{s}$ in terms of $\rho$, also as a bilinear transformation. In a slightly different form Eq. (14) reads

$$
r_{s}=\left[\rho-\cos 2\left(90^{\circ}-\phi\right)\right] /\left[1-\rho \cos 2\left(90^{\circ}-\phi\right)\right] .
$$

Equation (15) is identical in form with Eq. (13) and is obtained from it by switching the variables $r_{s}$ and $\rho$ and replacing $\phi$ by $90^{\circ}-\phi$. Consequently, Figs. 8-10 describe the inverse transformation $\rho \rightarrow r_{s}$ if, in these figures, we interchange $r_{s}$ and $\rho$ and replace $\phi$ by $90^{\circ}-\phi$.

\section{REFLECTION PHASE SHIFTS AND DIELECTRIC FUNCTION FROM MEASURED $p$ AND $S$ REFLECTANCES AT THE SAME ANGLE OF INCIDENCE}

In addition to providing new understanding of the behavior of the fundamental Fresnel reflection coefficients for the $p$ and $s$ polarizations, and also a unified framework for discussion of several specific reflection characteristics, this paper leads to other useful practical results. In particular, we provide simple analytical and graphical methods for determining reflection phase shifts and the dielectric function from measurements of the interface reflectances of the $p$ and $s$ polarizations. This problem has been dealt with before. ${ }^{14}$

The interface reflectances are defined by

$$
R=\left|r_{l}\right|^{2}, l=p, s .
$$

If we take the squared absolute value of both sides of Eq. (6), we obtain

$$
R_{p}=R_{s} \frac{R_{s}+\cos ^{2} 2 \phi-2 R_{s}^{1 / 2} \cos 2 \phi \cos \delta_{s}}{1+R_{s} \cos ^{2} 2 \phi-2 R_{s}^{1 / 2} \cos 2 \phi \cos \delta_{s}},
$$

where $\delta_{s}=\arg r_{s}$ is the reflection phase shift for the $s$ polarization. Equation (17) can be readily solved for $\delta_{s}$ :

$$
\cos \delta_{s}=\frac{\left(R_{s}^{2}-R_{p}\right)+R_{s}\left(1-R_{p}\right) \cos ^{2} 2 \phi}{2 R_{s}^{1 / 2}\left(R_{s}-R_{p}\right) \cos 2 \phi} .
$$

Equation (18) provides a simple explicit solution for the reflection phase shift $\delta_{s}$ in terms of the intensity reflectances $R_{p}$ and $R_{s}$ of the $p$ and $s$ polarizations at the same angle of incidence ${ }^{15} \phi$. Once $\delta_{s}$ has been found, the complex relative
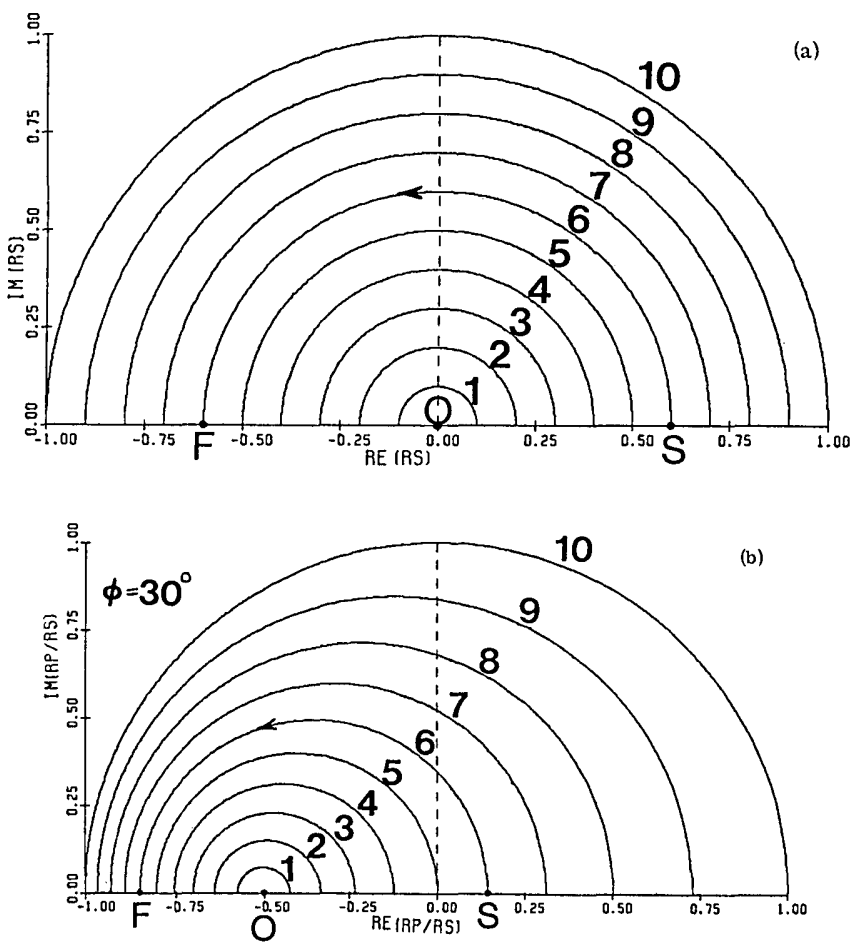

FIG. 9. Mapping of $r_{s}$ onto $\rho=r_{p} / r_{s}$ according to the bilinear transformation $\rho=\left(r_{s}-\cos 2 \phi\right) /\left(1-r_{s} \cos 2 \phi\right)$ when $\phi=30^{\circ}$. Semicircles centered on the origin in the $r_{s}$ plane, $\left|r_{s}\right|=0.1,0.2, \ldots, 1.0$, are mapped onto semicircles of coaxial circles that enclose the point $\rho=\cos 2 \phi$ in the $\rho$ plane. 

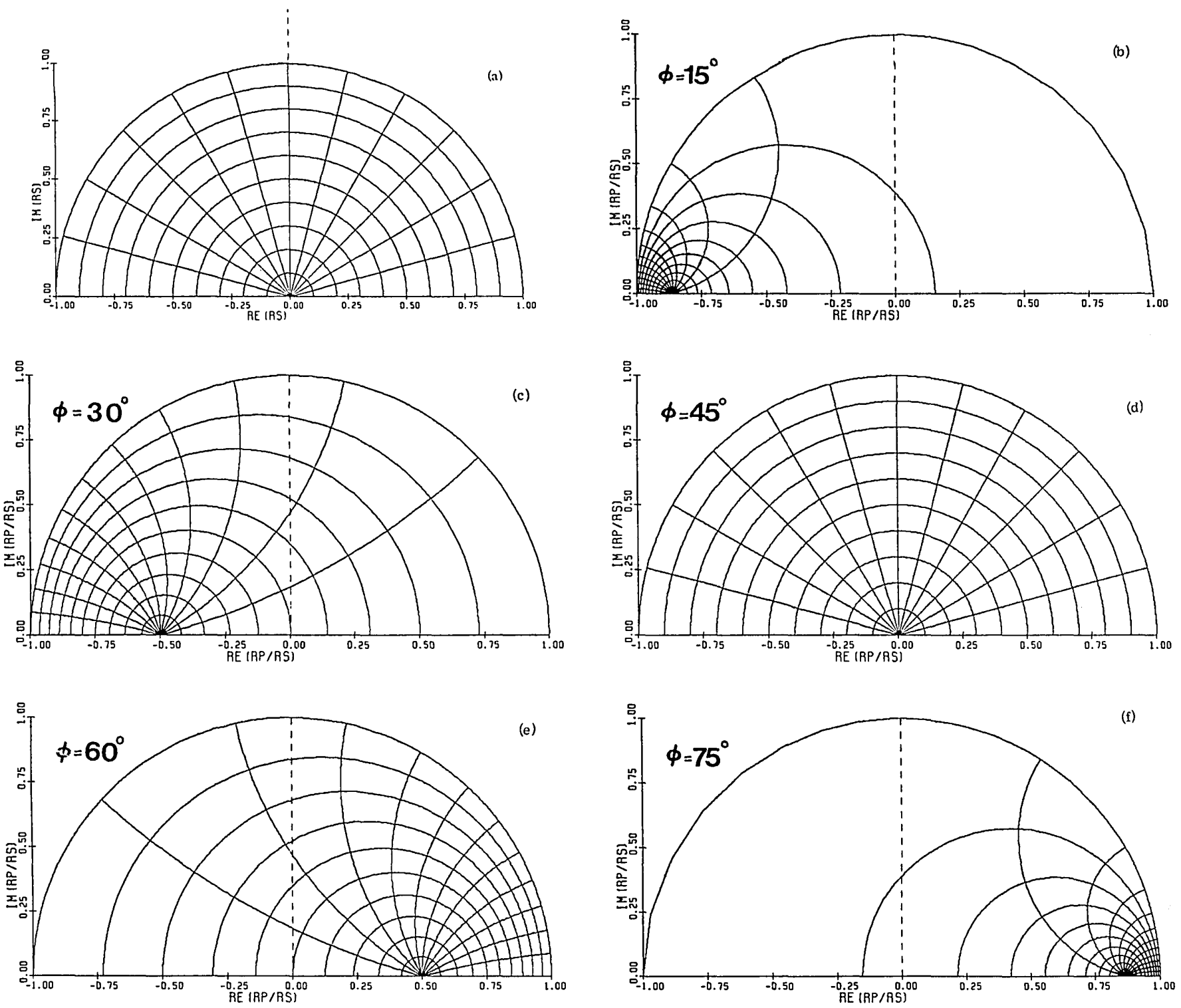

FIG. 10. Mapping of $r_{s}$ onto $\rho=r_{p} / r_{s}$ according to the bilinear transformation $\rho=\left(r_{s}-\cos 2 \phi\right) /\left(1-r_{s} \cos 2 \phi\right)$ when $\phi=15^{\circ}, 30^{\circ}, 45^{\circ}, 60^{\circ}, 75^{\circ}$. The orthogonal families of straight lines and semicircles through and around the origin in the $r_{s}$ are mapped onto orthogonal circle arcs and semicircles through and around the point $\rho=-\cos 2 \phi$ in the $\rho$ plane. The orthogonal sets that correspond to $\phi=30^{\circ}$ are obtained from the superposition of Figs. 8 and 9 . To identify individual curves use Figs. 8 and 9 as a guide.

dielectric function $\epsilon$ (the ratio of the dielectric function of the medium of refraction to that of the medium of incidence) can be determined from the now-known complex reflection coefficient $r_{s}=R_{s}{ }^{1 / 2} e^{j \delta_{s}}$ by

$$
\epsilon=\sin ^{2} \phi+\cos ^{2} \phi\left[\left(1-r_{s}\right) /\left(1+r_{s}\right)\right]^{2},
$$

which follows directly from Eq. (2).

If we take the argument (angle) of both sides of Eq. (13) or (6), we get

$$
\tan \Delta=\frac{R_{s}^{1 / 2} \sin \delta_{s} \sin ^{2} 2 \phi}{R_{s}^{1 / 2} \cos \delta_{s}\left(1+\cos ^{2} 2 \phi\right)-R_{s} \cos 2 \phi},
$$

where $\Delta=\arg \rho=\delta_{p}-\delta_{s}$. Equation (20) gives $\Delta$, hence $\delta_{p}$ $=\Delta+\delta_{s}$, after $\delta_{s}$ has been obtained from Eq. (18).

Simple nomograms can be constructed that provide graphical solutions for $\delta_{s}$ in terms of $R_{p}$ and $R_{s}$. Figure 11 shows one such nomogram in the $r_{s}$ plane for angle of incidence $\phi=60^{\circ}$. It consists of two intersecting sets of semicircles, one centered on the origin representing $\left|r_{s}\right|=$ constant, and the other encircling the point $r_{s}=\cos 2 \phi$ and representing $|\rho|=$ constant. The latter has already been mentioned in connection with Eq. (15). For given values of $R_{p}$ and $R_{s}$ we determine $\left|r_{s}\right|=R_{s}^{1 / 2}$ and $|\rho|=\left(R_{p} / R_{s}\right)^{1 / 2}$ and the point of intersection of the two semicircles that correspond to these values determine the complex reflection coefficient $r_{s}$. The angular polar coordinate of the point of intersection is $\delta_{s}$.

Instead of preprepared nomograms, one can also make one's own graphical construction to determine $\delta_{s}$ from $R_{p}$ and $R_{s}$ (or equivalently from $\left|r_{s}\right|$ and $|\rho|$ ). From Eq. (13) we write

$$
|\rho \cos 2 \phi|=\left|r_{s}-\cos 2 \phi\right| /\left|r_{s}-\sec 2 \phi\right| .
$$




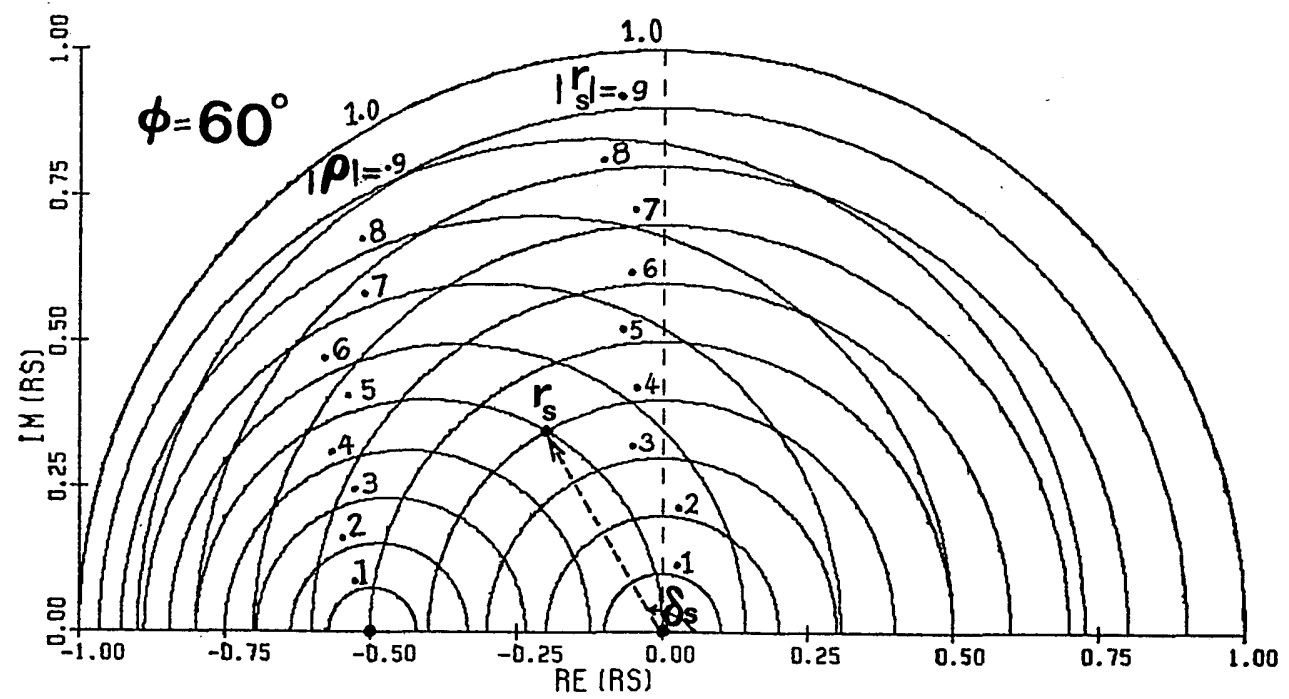

FIG. 11. A nomogram in the complex $r_{s}$ plane for the graphical determination of the reflection phase shift $\delta_{s}$ from the measured reflectances $R_{s}$ and $R_{p}$ of the $s$ and $p$ polarizations at the same angle of incidence $\phi=60^{\circ}$. The semicircles centered on the origin represent lines of equal amplitude reflectance for the $s$ polarization $\left|r_{s}\right|=R_{s}^{1 / 2}=0.1,0.2,0.3, \ldots, 1.0$, while the semicircles that enclose the point $r_{s}=\cos 2 \phi$ represent lines of equal ratio of $p$ and $s$ amplitude reflectances $|\rho|=\left(R_{p} / R_{s}\right)^{1 / 2}=0.1,0.2,0.3, \ldots, 1.0$. The measured reflectances $R_{s}$ and $R_{p}$ specify one semicircle from each family and their point of intersection gives the complex reflection coefficient $r_{s}$ while its angular polar coordinate gives $\delta_{s}=\arg r_{s}$.

According to Eq. (21), the circle in the $r_{s}$ plane that represents $|\rho|$ equal to a constant is recognized as the locus of a point $r_{s}$ that has the ratio of its distances to the two fixed points $\cos 2 \phi$ and $\sec 2 \phi$ on the real axis equal to a constant given by $|\rho| \times$ $|\cos 2 \phi|$. The following procedure to graphically determine $\delta_{s}$ becomes evident (see Fig. 12): (i) Mark the points $A$ and $B$ on the real axis at $\cos 2 \phi(O A)$ and $\sec 2 \phi(O B)$ respectively. (ii) Find the points $C$ and $D$ which internally and externally divide $A B$ in the same ratio $|\rho \cos 2 \phi|=\left(R_{p} / R_{s}\right)^{1 / 2}|\cos 2 \phi|$. (iii) Draw a semicircle with $C D$ as a diameter. (iv) Draw a second semicircle with the origin $O$ as center and of radius $\left|r_{s}\right|$ $=R_{s}^{1 / 2}$. (v) The point of intersection of the two semicircles $P$ gives the complex reflection coefficient $r_{s}$ and its angular polar coordinate gives $\delta_{s}$.

Similar nomograms and graphical constructions in the complex $\rho$ plane can be used to determine $\Delta$, as the reader can readily verify.

\section{APPENDIX}

We will prove that when $r_{p}$ is real, negative, and in the range

$$
-1 \leq r_{p} \leq-\tan ^{2}\left(\phi-45^{\circ}\right),
$$

$r_{s}$ becomes complex and its locus, for a given angle of incidence $\phi$, is an arc of a circle with center on the real axis at $\sec 2 \phi$ and radius of $|\tan 2 \phi|$.

It is evident from Eq. (11) that $r_{s}$ becomes complex when $r_{p}$ is real only if the quantity under the square root is negative. If we denote such a quality by $Q$,

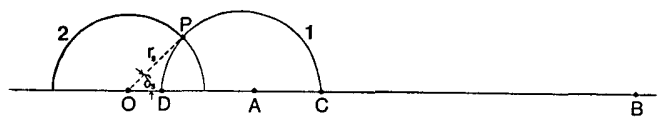

FIG. 12. A graphical construction that can be readily made and used in lieu of the nomogram of Fig. 11.

$$
Q=r_{p}+(1 / 4) \cos ^{2} 2 \phi\left(1-r_{p}\right)^{2},
$$

the range of $r_{p}$ values that make $Q$ negative lies between the roots of the quadratic equation

$$
Q=0 \text {. }
$$

These roots can be put in the form (after some trigonometry)

$$
r_{p}=-(\sec 2 \phi \mp \tan 2 \phi)^{2}=-\tan ^{2}\left(\phi \mp 45^{\circ}\right) .
$$

Of the range of $r_{p}$ values between the two roots of Eq. (25), only the subinterval specified by Eq. (22) is physically meaningful because $\left|r_{p}\right| \leq 1$.

With $Q$ negative, Eq. (11) can be rewritten as

$$
r_{s}=x+j y,
$$

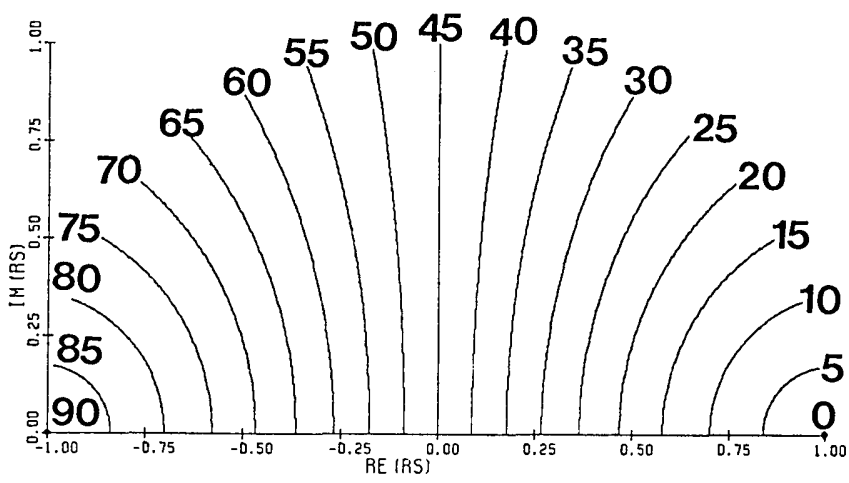

FIG. 13. The loci of the complex reflection coefficient for the $s$ polarization when the reflection coefficient for the $p$ polarization is real and negative in the range $-1 \leq r_{p} \leq-\tan ^{2}\left(\phi-45^{\circ}\right)$ at several different angles of incidence $\phi=0,5^{\circ}, 10^{\circ}, \ldots, 90^{\circ}$ marked by each curve. Each locus or curve at a given $\phi$ is an arc of a circle with center on the real axis at sec2 $\phi$ and radius of $|\tan 2 \phi|$. Circle arcs that correspond to $\phi=45^{\circ}+\theta$ and $45^{\circ}$ $-\theta$ are mirror images of one another with respect to the imaginary axis which represents $\phi=45^{\circ}$. 


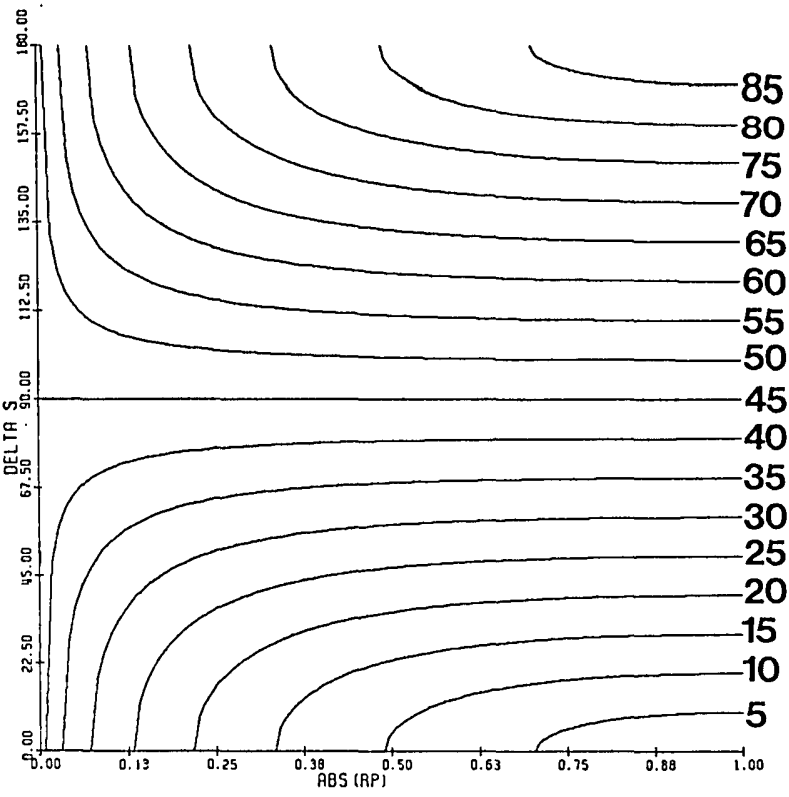

FIG. 14. The reflection phase shift for the $s$ polarization $\delta_{s}$, when the reflection phase shift for the $p$ polarization $\delta_{p}$ is equal to $\pi$, as a function of the absolute value of $r_{p}$, with the angle of incidence $\phi$ as a parameter marked by each curve. Mirror reflection with respect to the line $\delta_{s}=90^{\circ}$, which represents $\phi=45^{\circ}$, relate $\delta_{s}\left(\left|r_{p}\right|\right)$ at any pair of angles of incidence $\phi=45^{\circ} \pm \theta$.

where

$$
x=(1 / 2) \cos 2 \phi\left(1-r_{p}\right), y=(-Q)^{1 / 2} .
$$

Elimination of $r_{p}$ between $x$ and $y$ [where $Q$ is given by Eq. (23)] gives

$$
(x-\sec 2 \phi)^{2}+y^{2}=\tan ^{2} 2 \phi,
$$

which is the equation of a circle with center at $(\sec 2 \phi, 0)$ and radius of $|\tan 2 \phi|$. Of course $r_{s}$ is restricted to the arc of this circle between the real axis and the upper half of the unit circle. Figure 13 shows the locus of $r_{s}$, when $r_{p}$ is real, negative, and in the range specified by Eq. (22), for angles of incidence $\phi$ from $5^{\circ}$ to $85^{\circ}$ in steps of $5^{\circ}$. The limiting cases of $\phi=0$ and $\phi=90^{\circ}$ are represented by the points $r_{s}=1$ and $r_{s}$ $=-1$ respectively.

From Eqs. (26), (27), and (23), we obtain the interesting result $\left|r_{s}\right|^{2}=-r_{p}$, hence

$$
\left|r_{s}\right|=\left|r_{p}\right|^{1 / 2}
$$

Equation (29) indicates that the absolute value of the reflection coefficient (amplitude reflectance) for the $s$ polarization is equal to the square root of that for the $p$ polarization, when $r_{p}$ is real negative and $r_{s}$ is complex. From Eq. (29), the intensity reflectances are interrelated by

$$
R_{p}=R_{s}^{2},
$$

a relation that may have been thought before to hold only when the angle of incidence ${ }^{5-7}$ or the angle of refraction ${ }^{9}$ is $45^{\circ}$.

For completeness, Fig. 14 gives the reflection phase shift $\delta_{s}=\arg r_{s}$ as a function of $\left|r_{p}\right|$ for different angles of incidence $\phi$ from $5^{\circ}$ and $85^{\circ}$ in steps of $5^{\circ}$.

The results of this appendix complete an earlier analysis ${ }^{12}$ in which we proved, starting directly from the equations for the Fresnel coefficients, Eqs. (1) and (2), that $r_{p}$ can be real while $r_{s}$ is complex, and in which we also found the conditions that must be satisfied by the dielectric function $\epsilon$ for this to happen.

${ }^{1}$ See, for example, M. Born and E. Wolf, Principles of Optics (Pergamon, New York, 1975), 5th edition, p. 40.

${ }^{2} \mathrm{R}$. H. Muller, "Definitions and conventions in ellipsometry," Surf. Sci. 16, 14-33 (1969).

${ }^{3}$ R. M. A. Azzam, "Transformation of Fresnel's interface reflection and transmission coefficients between normal and oblique incidence," J. Opt. Soc. Am. 69, 590-596 (1979).

${ }^{4}$ Equation (6) can be cast in the alternative form $\left(r_{s}{ }^{2}-r_{p}\right) /\left(r_{s}-r_{s} r_{p}\right)$ $=\cos 2 \phi$. The left-hand-side function of $r_{s}$ and $r_{p}$ is therefore real and invariant, at a given angle of incidence $\phi$, with respect to changes of media and/or wavelength.

${ }^{5}$ R. M. A. Azzam, "On the reflection of light at $45^{\circ}$ angle of incidence," Opt. Acta 26 (1979), (in press).

${ }^{6} \mathrm{D}$. W. Berreman, "Simple relation between reflectances of polarized components of a beam when the angle of incidence is $45^{\circ}$," J. Opt. Soc. Am. 56, 1784 (1966).

7S. P. F. Humphreys-Owen, "Comparison of reflection methods for measuring optical constants without polarimetric analysis, and proposal for new methods based on the Brewster angle," Proc. Phys. Soc. Lond. 77, 949-957 (1961)

${ }^{8}$ The second root of the quadratic equation that results from setting the numerator of the right-hand side of Eq. (8) equal to zero has an absolute value greater than 1 , hence is physically unacceptable.

${ }^{9}$ R. M. A. Azzam, "Consequences of light reflection at the interface between two transparent media such that the angle of refraction is $45^{\circ}$," J. Opt. Soc. Am. 68, 1613-1615 (1978).

${ }^{10}$ See, for example, A. Kyrala, Applied Functions of a Complex Variable (Wiley-Interscience, New York, 1972), Ch. 8.

${ }^{11}$ The mapping is conformal at all points in the complex plane except where $\partial r_{p} / \partial r_{s}=0$ or $\infty$. This excludes the point specified by $r_{s}$ and $r_{p}$ in Eqs. (9) and (10).

${ }^{12}$ R. M. A. Azzam, "Reflection of an electromagnetic plane wave with 0 or $\pi$ phase shift at the surface of an absorbing medium," J. Opt. Soc. Am. 69, 487-488 (1979).

${ }^{13}$ R. M. A. Azzam and N. M. Bashara, Ellipsometry and polarized Light (North-Holland, Amsterdam, 1977).

${ }^{14}$ M. R. Querry, "Direct solution of the generalized Fresnel reflectance equations," J. Opt. Soc. Am. 59, 876-877 (1969).

${ }^{15}$ When $\phi=45^{\circ}$, we have $\cos 2 \phi=0$ and $R_{p}=R_{s}^{2}$. This reduces Eq. (18) to $\cos \delta_{s}=0 / 0$, hence $\delta_{s}$ becomes indeterminate, as is expected in this special case. 